Volume 14, No 3 International Journal of Radiation Research, July 2016

\title{
Dosimetric comparison between intensity modulated radiotherapy and three dimensional conformal radiotherapy planning in patients with locally advanced cervical carcinoma
}

\author{
A. Naik, O.P. Gurjar*, P. Bagdare, K.L. Gupta, V. Bhandari, M. Kausar, \\ D.D. Ladia, H.K. Mittal
}

Roentgen-SAIMS Radiation Oncology Centre, Sri Aurobindo Institute of Medical Sciences, Indore, India

\begin{abstract}
- Original article

\section{*Corresponding author:}

Dr. Om Prakash Gurjar,

E-mail:ominbarc@gmail.com

Revised: Sept. 2015

Accepted: Nov. 2015

Int. J. Radiat. Res., July 2016;

14(3): 189-196

DOI: 10.18869 /acadpub.ijrr.14.3.189

Background: External beam radiotherapy (EBRT) is mainstay of treatment in patients with locally advanced cervical carcinoma (LACC). Three dimensional conformal radiotherapy (3DCRT) and intensity modulated radiotherapy (IMRT) are mainly used in EBRT. However which one is superior is still controversial. Materials and Methods: Forty patients of LACC treated with IMRT were selected for this study. 3DCRT plans were also created for all the patients. 3DCRT and IMRT plans were compared on the basis of planning target volume (PTV) coverage, dose to normal organs, homogeneity index (HI) and conformity index $\left(\mathrm{Cl}_{95 \%}\right)$. Results: In both the techniques $99 \%$ of PTV was covered with more than $96 \%$ of prescribed dose (PD). $D_{15}, D_{35}$ and $D_{50}$ (Dose to $15 \%, 35 \%$ and $50 \%$ volume respectively) for bladder was reduced by $2.09 \%$, $14.623 \%$ and $32.57 \%$ and for the rectum it was reduced by $7.46 \%, 23.82 \%$ and $43.68 \%$ in IMRT compared to 3DCRT. $V_{45}$ (volume receiving $45 \mathrm{~Gy}$ ) in case of bowel were found to be much less in IMRT in comparison to 3DCRT. Insignificant difference found between doses to femoral heads in IMRT and 3DCRT. The $\mathrm{Cl}_{95 \%}$ in IMRT plans was found much better than that in 3DCRT whereas $\mathrm{HI}$ in both the techniques were found almost same. Conclusion: IMRT significantly reduced the irradiated volume of OAR and improved dose conformity in the PTV compared to that by 3DCRT. So, it can be concluded that IMRT should be chosen as preferred technique for the EBRT of LACC with proper immobilizing devices and imaging.
\end{abstract}

Keywords: External radiotherapy, IMRT, locally advanced cervical carcinoma, 3DCRT.

\section{INTRODUCTION}

Worldwide cervical cancer (Ca $\mathrm{Cx}$ ) remains the most common gynecologic cancer, with over 500,000 women globally develop $\mathrm{Ca} \mathrm{Cx}$ and 233,000 women dying of the disease every year majority of these mortality occurring in developing countries( 1 (1) With approximately 132,000 new women diagnosed and 74,000 deaths annually, India accounts for nearly one third of the global $\mathrm{Ca} \mathrm{Cx}$ deaths. Indian women face a $2.5 \%$ cumulative lifetime risk and $1.4 \%$ cumulative death risk from cervical cancer (2). The incidence of Ca Cx usually rises in 30-34 years of age and peaks at 55-65 years, with a median age of 38 years. Sexually transmitted human papilloma virus (HPV) infection is the most important risk factor for cervical intraepithelial neoplasia and invasive Ca Cx. HPV serotypes 16 and 18 account for nearly $76.7 \%$ of Ca Cx in India (3).

External beam radiotherapy (EBRT) along with Intracavitory Brachytherapy remains the mainstay of treatment in locally advanced 
carcinoma cervix (LACC) patients (4). Over the decades, the treatment fields and volumes for LACC have remained largely unchanged despite of various technological advances in radiotherapy delivery. In treatment of $\mathrm{Ca} \mathrm{Cx}$, it is important to deliver adequate dose not only to the primary tumor, but also to the pelvic lymph nodes to maximize tumor control. For LACC patients, the whole uterus, upper vagina, bilateral parametrial tissues, uterosacral ligaments and draining regional lymph nodes are treated in the standard radiotherapy practice. A very large portion of rectum, bladder and bowel are also included in the high dose region in order to encompass these target volumes in mostly available technique i.e. two dimensional radiotherapy (2DRT) and three dimensional conformal radiotherapy (3DCRT). This highlights a persistent problem in the treatment of $\mathrm{Ca} \mathrm{Cx}$ that in part reflects an inability to deliver sufficient dose to tumorbearing regions without exceeding critical normal tissue tolerances (5-7). The main aims of radiotherapy are to increase tumor control probability (TCP) and decrease the normal tissue complications probability (NTCP) and decision regarding the choice of treatment technique, beam placement and the imaging technique is made keeping these two principles in mind $(8,9)$. While 3DCRT, through the use of computed tomography (CT) imaging and planning, allows for greater normal tissue sparing, the field arrangements have remained largely unchanged. Thus recent advances are focused towards delivering radiation to target volumes without exceeding normal tissues tolerances by using more conformal techniques leading to development of new modern radiotherapy techniques such as intensity modulated radiotherapy (IMRT). IMRT by using numerous radiation beams with optimized intensity modulation within the field and high dose gradient around the target can effectively distribute the radiation dose homogenously throughout the target volume sparing surrounding normal structures. IMRT can thus achieve much better dose conformity than 3DCRT. However various studies have shown inconsistent finding in regard to normal organ

Int. J. Radiat. Res., Vol. 14 No. 3, July 2016 sparing by IMRT as compared to 3DCRT for definitive treatment of LACC.

Hence this study aims to compare dosimetric aspects of 3DCRT and IMRT on the basis of target coverage and doses to organs at risk (OAR).

\section{MATERIALS AND METHODS}

One hundred twelve patients of $\mathrm{Ca} \mathrm{Cx}$ were referred for definitive radiotherapy in our institute from January 2014 to May 2015, out of which forty patients of LACC (FIGO stage IIA to IVA)(10) treated with IMRT were selected for this retrospective study. An inform consent was taken from all the patients.

Patients with performance status or Karnofsky performance status score (KPS) of more than 60, all previously untreated patient with histologically proven squamous cell carcinoma cervix FIGO stage IIA to IVA were taken up for this study. Patients who had already received treatment either in form of radiotherapy or had undergone any form of hysterectomy, patients with other comorbidities, and pregnant patients were excluded. Patient characteristics are given in table 1.

Table 1. Characteristics of study population.

\begin{tabular}{|l|l|}
\hline No. of Patients & 40 \\
\hline Age (Years) & Range: 30-75; Mean: 52 \\
\hline Gender & Female \\
\hline Tumor site & Cervix \\
\hline Histology & Squamous cell carcinoma \\
\hline FIGO stage & II A: 03 \\
& II B :08 \\
& III A:08 \\
& III B : 19 \\
& IV A :02 \\
\hline
\end{tabular}

\section{CT Simulation}

All the patients were immobilized in supine position using pelvic base plate (POCL Pvt. Ltd., Mumbai) and thermoplastic sheet (Orfit). Siemens SOMATOM Definition AS scanner (Siemens Medical Systems, Erlangen, Germany) was utilized for the CT simulation of these patients. CT images of $3 \mathrm{~mm}$ slice thickness were 
obtained of the region including whole abdomen and pelvis. These images were transferred to the treatment planning system (TPS) Eclipse vs. 8.9 (Varian Medical systems, Palo Alto, CA) for radiotherapy planning.

\section{Target and $\mathrm{OAR}$ delineation}

Gross tumor volume (GTV), Clinical Target Volume (CTV), Planning Target Volume (PTV) and OAR like bladder, rectum, bowel bag, femoral heads rectosigmoid etc. were delineated on the CT image of all the patients by same radiation oncologist to decrease interpersonal differences. RTOG Guidelines were used for delineation of target volumes and OAR. While contouring GTV consisted gross visible tumor and its visible extension and CTV consists GTV, uterine cervix, uterine corpus, parametrium, vagina and ovaries includes involved nodes and relevant draining nodal groups (common iliac, internal iliac, external iliac, obturator and presacral Lymph Node). PTV include a generous margins of $1 \mathrm{~cm}$ around the CTV in region the uterus and cervix to account for uterine motion and any setup errors and of $0.5 \mathrm{~cm}$ in the nodal regions (11).

\section{Treatment planning}

All the plans were planned on Eclipse TPS for the dose of 50 Gray (Gy) in 25 fractions (\#) with 5\# / weeks schedule. This TPS is commissioned for the medical electron linear accelerator (Clinac) Varian Clinac DMX (Varian Medical systems, Palo Alto, CA) having 6 and 15 mega voltage (MV) photon energies and 6, 9, 12 and 15 mega electron volt $(\mathrm{MeV})$ electron energies. The Clinac is equipped with Millennium 80 multi leaf collimator (MLC) system having 40 pairs of leaves, each leaf having a width of $1 \mathrm{~cm}$ projected at isocentre. The MLC leaf ends are rounded. Tongue and groove arrangement of leaves minimizes the interleaf leakage. The standard MLC leaf speed in dynamic window treatment mode is $2.5 \mathrm{~cm} / \mathrm{s}$.

\section{IMRT Plans}

IMRT plans were done using 6 MV energy with seven to nine coplanar (CP) gantry angles with couch angle $0^{\circ}$ and no parallel opposed fields were chosen. The isocentre was placed at 191 the geometrical centre of the PTV. The target volume in all the cases was large, so to cover the target volume wherever the field exceeded 14.5 $\mathrm{cm}$ in $\mathrm{x}$-direction, it got split in two fields automatically. The splitting is because of the system limitation which has the small field IMRT license only. The maximum distance between the most retracted and extended MLC cannot be more than $14.5 \mathrm{~cm}$. So, two fields at same gantry angle were used for most of the gantry angles in all the plans. In this way maximum of eighteen fields at nine gantry angles were used in some plans. Target volumes (PTV, CTV and GTV) and OAR were given the dose constraints (as shown in table 2) and adequate weights. Varian leaf motion calculator vs. 8.9.08 was used to calculate leaf motion for dynamic dose delivery. Plans were optimized by using dose volume optimizer (DVO). Analytical anisotropic algorithm (AAA) was utilized to calculate doses with grid size of $0.25 \mathrm{cc}$. Figure 1 (a) shows the field arrangement in one of the IMRT plan.

\section{DCRT Plans}

Four CP fields (AP-PA and two lateral fields) with couch angle $0^{\circ}$ were used in all the 3DCRT plans. The isocenter was placed at the geometrical centre of the PTV. The mean isocentre depth was $14.8 \mathrm{~cm}$ from lateral side and $9.67 \mathrm{~cm}$ from anterior - posterior side in the cases chosen for this study. So, to minimize the skin dose and for better PTV coverage, all the plans were done by using $15 \mathrm{MV}$ photon energy. The MLC leaves were fit to the PTV with $0.8 \mathrm{~cm}$ margin. Doses were calculated by using AAA with $0.25 \mathrm{cc}$ grid size. Weight of particular fields were decreased / increased by changing monitoring units (MU) wherever required to manage hot / cold spot and dose homogeneity.

Table 2. Dose constraints used for IMRT planning.

\begin{tabular}{|l|l|}
\hline Target and organs at risk & \multicolumn{1}{c|}{ Constraints } \\
\hline PTV & $\begin{array}{l}\mathrm{D}_{95}>97 \% \text { of PD } \\
\mathrm{D}_{\max }(\% \text { of PD) }<115 \% \text { of PD }\end{array}$ \\
\hline Bladder & $\mathrm{V}_{45} \leq 35 \%$ \\
\hline Rectum & $\mathrm{V}_{40} \leq 60 \%$ \\
\hline Femoral heads & $\mathrm{D}_{\max } \leq 50 \mathrm{~Gy}$ \\
\hline Bowel & $\mathrm{V}_{45} \leq 195 \mathrm{cC}$ \\
\hline
\end{tabular}

Int. J. Radiat. Res., Vol. 14 No. 3, July 2016 
Figure 1 (b) shows the field arrangement in one of the 3DCRT plan.

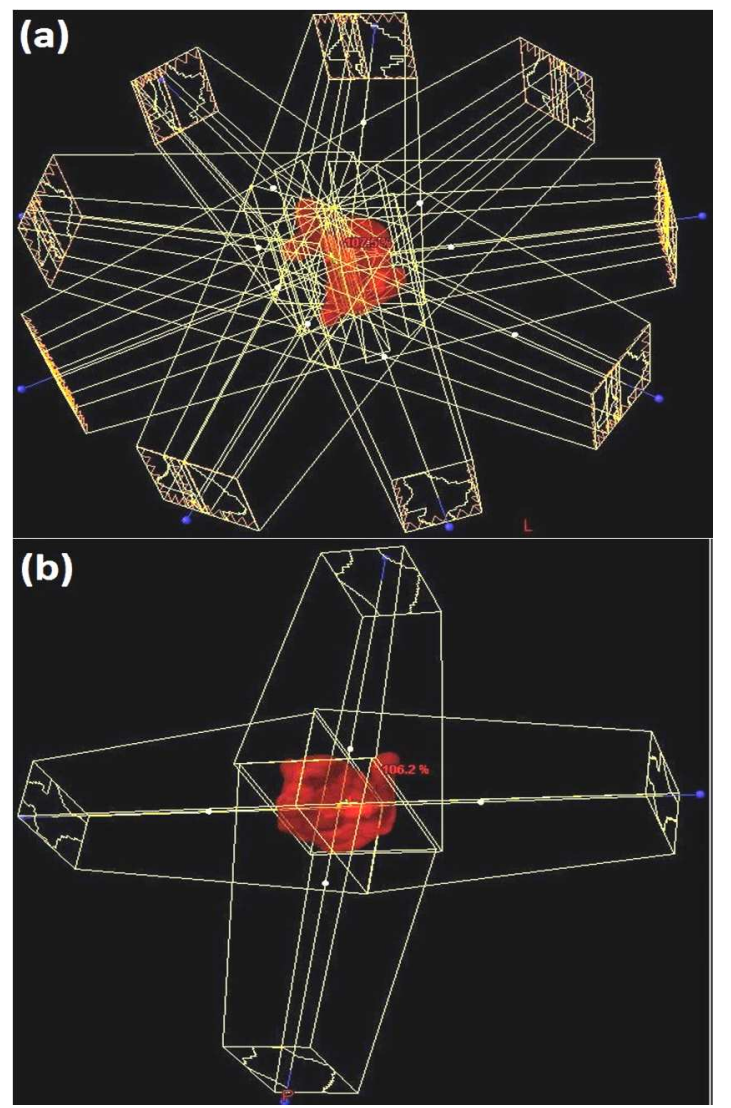

Figure 1. Field arrangement in (a) IMRT and (b) 3DCRT plan in one of the cases.

\section{Plan evaluation}

After approval, all the plans were exported to Clinac for treatment delivery. Dosimetric parameters of all the plans generated by both the techniques were compared objectively using the dose volume histograms (DVH).

Both kind of plans were compared for PTV coverage on the basis of $\mathrm{D}_{95}$ and $\mathrm{D}_{99}$ (Dose to 95\% and $99 \%$ PTV respectively) and $\mathrm{D}_{\max }$ (Maximum dose). Doses to OAR were also compared in both the techniques. For bladder and rectum values of $\mathrm{D}_{15}, \mathrm{D}_{35}$ and $\mathrm{D}_{50}$ (dose to $15 \%, 35 \%$ and $50 \%$ of organ volume); femoral heads, values of $D_{\max }$; bowel(small and large intestine) $\mathrm{V}_{45 \mathrm{~Gy}}$ (volume receiving $45 \mathrm{~Gy}$ ). Also, homogeneity index (HI) and conformity index (CI) for $95 \%$ of PD is calculated for both the techniques.

Int. J. Radiat. Res., Vol. 14 No. 3, July 2016
The HI and CI were calculated according to the formulae given below (12);

CI $I_{95 \%}=$ Total volume receiving 95\% of PD / PTV

$\mathrm{HI}_{95 \%}=\mathrm{D}_{5} / \mathrm{D}_{95}$; where, $\mathrm{D}_{5}$ and $\mathrm{D}_{95}$ are the doses received by $5 \%$ and $95 \%$ of PTV.

The value of $\mathrm{CI}=1.0$ (one) and $\mathrm{HI}=1.0$ (one) is considered the ideal.

The statistical significant difference between each set of dosimetric parameters was known by calculating $p$-value using student's $t$-test. A value of $<0.05$ was considered significant.

\section{RESULTS}

The mean coverage for 95\% of PTV was $98.8 \%$ and $99.86 \%$ of PD in 3DCRT and IMRT plans respectively. Also, the mean coverage for $99 \%$ of PTV was $97.18 \%$ and $96.22 \%$ of PD in 3DCRT and IMRT plans respectively. So, the target coverage in all the plans was deemed acceptable. The mean $\mathrm{D}_{\max }$ for PTV was $105.28 \%$ (range 104.24 - 108.2\%) for 3DCRT plans and $106.73 \%$ (range 103.80 - 108.64\%) for IMRT plans.

The mean CI was found to be 2.18 and 1.462 in the plans by 3DCRT and IMRT respectively, the difference between the $\mathrm{CI}$ in both the techniques was extremely significant with the $p$ value 0.0001 . The mean $\mathrm{HI}$ was found to be 1.055 and 1.073 in the plans by 3DCRT and IMRT respectively, the difference between the HI in both the techniques was non-significant with the $p$-value 0.2042 . The detailed results of target coverage, $\mathrm{HI}$ and $\mathrm{CI}$ in all the plans by both the techniques are given in table 3.

Table 3. Mean values of PTV coverage, $\mathrm{HI}$ and $\mathrm{Cl}$ 3DCRT and IMRT plans of forty patients.

\begin{tabular}{|cc|c|c|c|}
\hline \multicolumn{2}{|c|}{$\begin{array}{c}\text { Dosimetric } \\
\text { Parameters }\end{array}$} & \multicolumn{1}{|c|}{ IMRT } & \multicolumn{1}{|c|}{ 3DCRT } & $\begin{array}{c}p- \\
\text { Values }\end{array}$ \\
\hline PTV & & & & \\
& $\mathrm{D}_{95}(\%$ of PD) & $99.86 \pm 1.44$ & $98.80 \pm 1.76$ & 0.0041 \\
& $\mathrm{D}_{99}(\%$ of PD) & $96.22 \pm 1.85$ & $97.19 \pm 1.81$ & 0.0209 \\
& $\mathrm{D}_{\max }(\%$ of PD) & $106.73 \pm 1.40$ & $105.28 \pm 0.92$ & 0.0098 \\
\hline $\mathrm{HI}$ & & $1.074 \pm 0.088$ & $1.055 \pm 0.0159$ & 0.2042 \\
\hline $\mathrm{Cl}$ & & $1.462 \pm 0.207$ & $2.183 \pm 0.317$ & 0.0001 \\
\hline
\end{tabular}

$D_{x}(\%$ of $P D)=\%$ of $P D$ to $X \%$ of PTV; Dmax (\% of PD) = maximum dose in \% of PD; $\mathrm{HI}=$ Homogeneity index; $\mathrm{Cl}=$ Conformity Index 
The doses to bladder were reduced by $2.09 \%$ $\left(\mathrm{D}_{15} ; p=0.0001\right), 14.623 \%\left(\mathrm{D}_{35} ; p=0.0001\right)$ and $32.57 \%\left(\mathrm{D}_{50} ; p=0.0001\right)$ and the doses to rectum were reduced by $7.46 \%\left(\mathrm{D}_{15} ; p=0.0092\right), 23.82 \%$ $\left(\mathrm{D}_{35} ; p=0.0001\right)$ and $43.68 \%\left(\mathrm{D}_{50} ; p=0.0001\right)$ in IMRT compared to 3DCRT. The mean $\mathrm{V}_{45}$ of bowel was $227.781 \mathrm{cc}$ and $132.018 \mathrm{cc}$ in 3DCRT and IMRT plans respectively which showed significant $(p$-value $=0.0001)$ reduction in average irradiated bowel volumes. The mean $\mathrm{D}_{\max }$ values for right and left femoral head were 50.80Gy and 50.67Gy in 3DCRT plans and 50.095Gy and 49.82Gy in IMRT plans, so the difference between the $D_{\text {max }}$ of both the femoral heads in both the techniques is not statistically significant. The detailed results of the doses to OAR are mentioned in table 4. The composite DVH of IMRT and 3DCRT plan of one of the patients is shown in figure 2 .

Table 4. Mean doses to organs at risk (OAR).

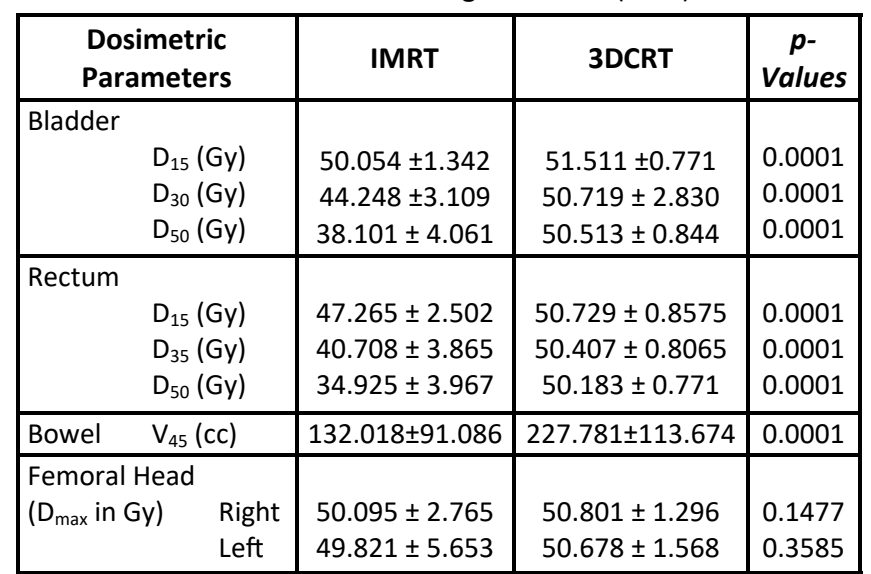

$D_{\mathrm{x}}$ : Dose to $\mathrm{x} \%$ of volume; $\mathrm{V}_{45}$ : Volume receiving $45 \mathrm{~Gy} ; \mathrm{D}_{\max }$ : maximum dose.

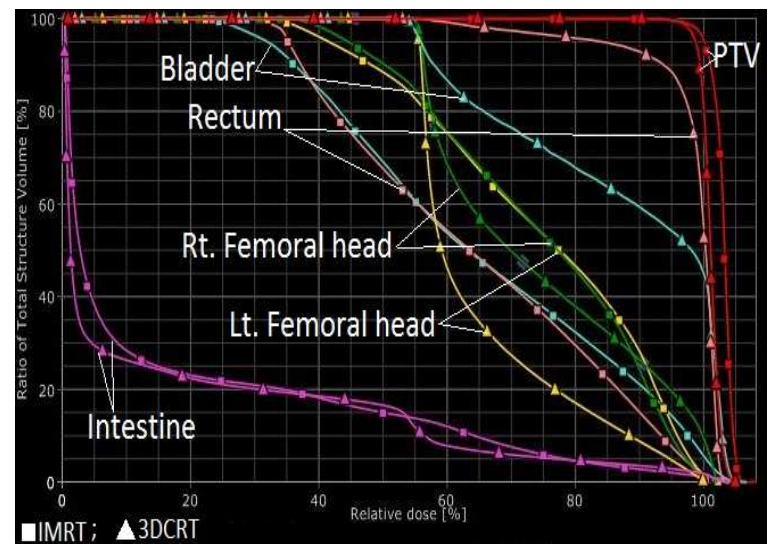

Figure 2. Composite dose volume histogram (DVH) of IMRT and 3DCRT plan for one of the patients.

\section{DISCUSSION}

Desired target coverage was achieved by both the techniques; $95 \%$ of PTV received more than $98 \%$ of PD with acceptable hotspot in all the plans by both the techniques. However, IMRT plans had significantly better PTV coverage and CI in comparison to that in 3DCRT.

Better coverage and $\mathrm{CI}$ in the case of IMRT plans was mainly because of the use of multiple beam angles and computer optimized intensity modulated beams. During optimization these beams are divided into several small beamlets, intensity of these beamlets is then modified using multileaf collimator (MLC) until desired dose distribution is achieved. This ability of IMRT to spatially and temporally modify the beam fluence introduces additional degrees of freedom for treatment planning and delivery in addition to field size shape and position. These intensity modulated beams after entering into patients body results in highly conformal dose distributions with complex target volume and thus sparing normal surrounding organs as shown in figure $3(\mathrm{a})$.

In 3DCRT, only four fields with uniform intensity were planned, thus the OAR before and after PTV in the path of each field also received the dose. The intersection of all the four fields makes rectangular shape (cross sectional view) where uniform dose from the entire four fields is received. This area covers not only irregular shaped PTV but also the OAR surrounding the PTV which results in high dose to OAR, this results in poor conformity of the dose around PTV as shown in figure 3 (b).

In present study, IMRT offered advantage over 3DCRT in terms of reduced dose to OAR especially the doses received by bladder rectum and bowel, but doses to femoral heads were found to be almost similar to that seen in 3DCRT. Results of this study concurred with the results of studies published already (13-16).

Van De Bunt et al.(16) compared conventional, conformal and IMRT plans for $\mathrm{Ca} \mathrm{Cx}$ and found that IMRT is superior in sparing of critical organs compared with conventional and conformal treatment, with adequate coverage of the target volumes and also noted that IMRT

Int. J. Radiat. Res., Vol. 14 No. 3, July 2016 
remains superior after 30 Gy external beam radiation therapy, despite tumor regression and internal organ motion. Recently in study done by Forrest et al.(17) comparing intensity modulated whole pelvis RT (IM-WPRT) with 4 Field conformal pelvic radiotherapy (4F-WPRT) for definitive management of Ca Cx showed a single, initial IM-WPRT plan with appropriate margins encompassing initial gross and potential microscopic pelvic disease leads a significant reduction in the dose to OAR at the $\mathrm{V}_{50}, \mathrm{~V}_{45}, \mathrm{~V}_{40}$ and $\mathrm{V}_{30}$ level with difference of $84 \%$ for bladder, $58 \%$ for small bowel, $54 \%$ for sigmoid and $84 \%$ in rectum for $\mathrm{V}_{50}$ in most patients without compromising target coverage with mean target volume covered by $95 \%$ dose was $99.7 \%$ for $4 \mathrm{~F}$ WPRT and $98.8 \%$ for IM-WPRT.

Mell et al.(18) compared IMRT, 3DCRT, and anterior-posterior parallel opposed pair with concurrent chemotherapy for treatment of $\mathrm{Ca} \mathrm{Cx}$ and found that IMRT reduced doses to the bone marrow and small bowel but the reduction to the rectum and bladder was less impressive. Similarly, Yang et al.(19) in their meta-analysis of 13 dosimetric studies comparing 3DCRT and IMRT treatment plans reported a $17.3 \%$ reduction in volume of the small bowel receiving 45 Gy and a 39.5\% reduction in rectal volumes receiving 45 Gy. No statistically significant decreases in bladder dose and bone marrow volumes with IMRT seen. While in the present study, significant dose reduction for rectum and bladder was observed in IMRT plans as compared to that in 3DCRT plans.

The decreased doses to OAR by using IMRT allows high dose (up to 66 - 70 Gy) delivery to boost central target volume in patients whom brachytherapy is not possible, which is not possible by 3DCRT.

It is assumed that the reduction in dose to OAR may confer a clinical benefit in terms of reduced toxicities at the OAR. A number of Clinical studies comparing IMRT and 3DCRT in treatment of LACC showed significantly lower toxicity at organs at risk in IMRT treated patients (20-22). Mundt et al.(20) in their study found that IMRT achieved lower rates of chronic GI toxicity at $11.1 \%$, compared to $50.0 \%$ for conventional RT. Compared with patients who received conventional RT, patients who received IMRT experienced fewer grade $1(30 \%$ vs. $8.3 \%), 2$ (16.7\% vs. $2.8 \%$ ), and 3 (3.3\% vs. $0 \%)$ toxicities. A recently published clinical trial from Ghandi et al.(24) noted significantly fewer grade 2 acute and grade 3 gastrointestinal toxicities with similar rates of hematological toxicity in IMWPRT than patients receiving WP-CRT in their study.

Brixey et al.(23) has shown in their study that rates of grade of toxicity for neutrophil count (ANC), and hemoglobin were similar between patients receiving IMRT and four-field WPRT without chemotherapy. However on addition of chemotherapy, patients receiving conventional WPRT experienced higher rates of grade $\geq 2$ WBC toxicity ( $60.0 \%$ vs. $31.2 \%$ ), and lower WBC (2.8 vs. $3.6 \mathrm{ug} / \mathrm{dL})$ and ANC (1874 vs. 2669) counts compared to patients receiving IMRT. IMRT also achieved a $27.5 \%$ reduction in patients requiring holding of scheduled chemotherapy doses (23).

Due to highly conformal dose delivery in

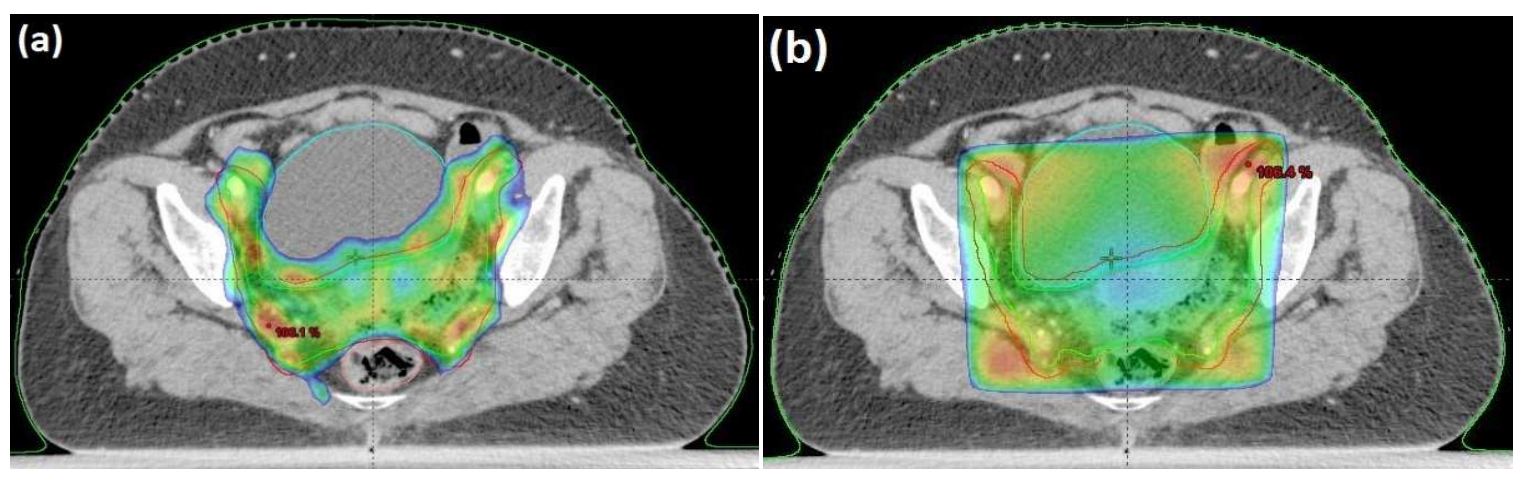

Figure 3. Dose wash ( $98 \%$ of prescribed dose) on same CT image in (a) IMRT and (b) 3DCRT plan. 
IMRT, it is of paramount importance to accurately delineate the target volumes which need to be irradiated to prevent under dosing and to protect OAR in near vicinity. This is particularly important in patients of $\mathrm{Ca} \mathrm{Cx}$ as these patients demonstrate more substantial amount of organ motion, tumor regression and deformation than in prostate cancer (16, 25-27). Several studies have shown the importance of cervical tumor regression during treatment, with a median volume reduction of $46 \%(16,27)$. This suggests that IMRT should be re-planned during the final third of treatment to take advantage of the shrinking GTV. Van De Bunt et al. (16) found repeated IMRT planning can improve the sparing of the bowel and rectum in patients with substantial tumor regression.

Bladder, rectum and small bowel are dynamic mobile structures that affect the position of the GTV (28-29). Protocols requiring a full bladder and empty rectum can minimize utero-cervical movement; such requirements are included in recent RTOG protocols [30]. As per 2011 RTOG consensus guideline for delineation of clinical target volume for intensity modulated radiotherapy for the definitive treatment of $\mathrm{Ca}$ Cx recommends a margins of $1.5-2 \mathrm{~cm}$ around the CTV in region of uterus and cervix and a PTV margin of $7 \mathrm{~mm}$ around the nodal CTV. However if soft tissue verification imaging modalities are not available then more generous margins must be used which might lead increased doses to OAR (10). These guidelines have been followed in the current study

One of the limitations of present study is that the peritoneal cavity has been contoured instead of outlining specific bowel loops. Since the probability of small bowel residing in a specific region of the pelvis is variable from day to day this type of contouring might overestimate the dose to bowel (31).

\section{CONCLUSION}

IMRT appears to offer several advantages over 3DCRT planning for definitive radiotherapy of LACC patients. These include a significant reduction in irradiated volume for bladder, 195 rectum and bowel, along with more conformal dose distribution in the PTV. It is anticipated that this would translate to overall reduction in acute and potentially late treatment-related toxicity.

\section{ACKNOWLEDGMENT}

We acknowledge our thanks to Chairman, SAIMS, Indore and Directors, Roentgen Oncology Solutions Pvt. Ltd. for allowing and encouraging us in conducting this study.

\section{Conflict of interest: Declared None}

\section{REFERENCES}

1. Barakat R, Markman M, Randall M (2009) Principles and practice of gynecologic oncology, 5th ed. Philadelphia: Lippincott Williams \& Wilkins.

2. Nandakumar A, Ramnath T, Chaturvedi M (2009) The magnitude of cancer cervix in India. Indian J Med Res, 130: 219-21.

3. Schiffman M, Castle PE, Jeronim J, Rodrigue AC, Wacholde $S$ (2007) Human papillomavirus and cervical cancer. Lancet, 370: 890-907.

4. Chemoradiotherapy for Cervical Cancer Meta-Analysis Collaboration (2008) Reducing uncertainties about the effects of chemoradiotherapy for cervical cancer: a systematic review and meta-analysis of individual patient data from 18 randomized trials. J Clin Oncol, 26: 5802-12.

5. Kirwan JM, Symonds P, Green JA, Tierney J, Collingwood M, Williams CJ (2003) A systemic review of acute and late toxicity of concomitant chemoradiation for cervical cancer. Radiother Oncol, 68: 217-26.

6. Eifel PJ, Levenback C, Wharton T, Oswald MJ (1995) Time course and incidence of late complications in patients treated with radiation therapy for FIGO stage IB carcinoma of the uterine cervix. Int J Radiat Oncol Biol Phys, 32: 12891300.

7. Forrest JL, Ackerman I, Barbera L, Barnes EA, Davidson $M$, Kiss A, et al. (2010) Patient outcome study of concurrent chemoradiation, external beam radiotherapy, and highdose rate brachytherapy in locally advanced carcinoma of the cervix. Int J Gynecol Cancer, 20: 1074-8.

8. Hunt MA, Schultheiss TE, Desobry GE, Hakki M, Hanks GE (1995) An evaluation of setup uncertainties for patients treated to pelvic sites. Int J Radiat Oncol Biol Phys, 32: 22733.

9. Gurjar OP, Mishra SP, Bhandari V, Pathak P, Pant S, Patel P (2014) A study on the necessity of kV-CBCT imaging compared to $\mathrm{kV}$-Orthogonal portal imaging based on setup

Int. J. Radiat. Res., Vol. 14 No. 3, July 2016 
errors: Considering other socioeconomical factors. J Can Res Ther, 10: 583-6.

10. Pecorelli S, Zigliani L, Odicino F (2009) Revised FIGO staging for carcinoma of the cervix. Int J Gynaecol Obstet, 105: 107 $-8$.

11. Lim K, Small W Jr, Portelance L, Creutzberg C, JürgenliemkSchulz IM, Mundt A, et al. (2011) Consensus guidelines for delineation of clinical target volume for intensitymodulated pelvic radiotherapy for the definitive treatment of cervix cancer. Int J Radiat Oncol Biol Phys, 79: 348-55.

12. Gurjar OP and Mishra SP (2013) Dosimetric analysis of intensity modulated radiotherapy plans having one or more pairs of parallel opposed beams among the set of beams in some special cases. Radiat Prot Environ, 36: 13842.

13. Portelance L, Chao KS, Grigsby PW, Bennet H, Low D (2001) Intensity-modulated radiation therapy (IMRT) reduces small bowel, rectum, and bladder doses in patients with cervical cancer receiving pelvic and para-aortic irradiation. Int J Radiat Oncol Biol Phys, 51: 261-6.

14. Heron DE, Gerszten K, Selvaraj RN, King GC, Sonnik D, Gallion $\mathrm{H}$, et al. (2003) Conventional 3D conformal versus intensity-modulated radiotherapy for the adjuvant treatment of gynecologic malignancies: a comparative dosimetric study of dose-volume histograms small star, filled. Gynecol Oncol, 91: 39-45.

15. Roeske JC, Lujan A, Rotmensch J, Waggoner SE, Yamada D, Mundt AJ (2000) Intensity-modulated whole pelvic radiation therapy in patients with gynecologic malignancies. Int J Radiat Oncol Biol Phys, 48: 1613-21.

16. Van de Bunt $L$, van der Heide UA, Ketelaars M, de Kort GA, Jurgenliemk-Schulz IM (2006) Conventional, conformal, andintensity-modulated radiation therapy treatment planning ofexternal beam radiotherapy for cervical cancer: the impact of tumor regression. Int J Radiat Oncol Biol Phys, 64: 189-96.

17. Forrest J, Presutti J, Davidson M, Hamilton P, Kiss A, Thomas G (2012) A Dosimetric Planning study comparing intensity-modulated radiotherapy with four-field conformal pelvic radiotherapy for the definitive treatment of cervical carcinoma. Clin Oncol (R Coll Radiol) 24: 63-70.

18. Mell LK, Tiryaki H, Ahn KH, Mundt AJ, Roeske JC, Aydogan B (2008) Dosimetric comparison of bone marrow-sparing intensity-modulated radiotherapy versus conventional techniques for treatment of cervical cancer. Int J Radiat Oncol Biol Phys, 71: 1504-10.

19. Yang B, Zhu L, Cheng H, Li Q, Zhang Y, Zhao Y (2012) Dosimetric comparison of intensity modulated radiotherapy and three-dimensional conformal radiotherapy in patients with gynecologic malignancies: a systematic review and meta-analysis. Radiat Oncol, 7: 197.

20. Mundt AJ, Mell LK, Roeske JC (2003) Preliminary analysis of chronic gastrointestinal toxicity in gynecology patients treated with intensity modulated whole pelvic radiation therapy. Int J Radiat Oncol Biol Phys, 56: 1354-60.
21. Mundt AJ, Roeske JC, Lujan AE, Yamada SD, Waggoner SE, Fleming G, Rotmensch J (2001) Initial clinical experience with intensity-modulated whole-pelvis radiation therapy in women with gynecologic malignancies. Gynecol Oncol, 82: 456-63.

22. Salama JK, Mundt AJ, Roeske J, Mehta N (2006) Preliminary outcome and toxicity report of extended-field, intensitymodulated radiation therapy for gynecologic malignancies. Int J Radiat Oncol Biol Phys, 65: 1170-76.

23. Brixey CJ, Roeske JC, Lujan AE, Yamada SD, Rotmensch J, Mundt AJ (2002) Impact of intensity-modulated radiotherapy on acute hematologictoxicity in women with gynecologic malignancies. Int J Radiat Oncol Biol Phys, 54: 1388-96.

24. Gandhi AK, Sharma DN, Rath GK, Julka PK, Subramani V, SharmaS, et al. (2013) Early clinical outcomes and toxicity of intensity modulated versus conventional pelvic radiation therapy for locally advanced cervix carcinoma: a prospective randomized study. Int J Radiat Oncol Biol Phys, 87: 542-8.

25. Buchali A, Koswig S, Dinges S, Rosenthal P, Salk J, Lackner $G$, et al. (1999) Impact of the filling status of the bladder and rectum on their integral dose distribution and the movement of the uterus in the treatment planning of gynaecological cancer. Radiother Oncol, 52: 29-34.

26. Chan $P$, Dinniwell $R$, Haider MA, Cho $Y B$, Jaffray $D$, Lockwood G, et al. (2008) Inter- and intrafractional tumour and organ movement in patients with cervical cancer undergoing radiotherapy: a cinematic- MRI point-of -interest study. Int J Radiat Oncol Biol Phys, 70: 1507-15.

27. Huh SJ, Park W, Han Y (2004) Interfractional variation in position of the uterus during radical radiotherapy for cervical cancer. Radiother Oncol, 71: 73-9.

28. Ahmad R, Hoogeman MS, Bondar M, Dhawtal V, Quint $S$, De Pree I, et al. (2011) Increasing treatment accuracy for cervical cancer patients using correlations between bladder-filling change and cervix-uterus displacements: proof of principle. Radiother Oncol 98: 340-6.

29. Bondar L, Hoogeman M, Mens JW, Dhawtal G, de Pree I, Ahmad R, et al. (2011)Toward an individualized target motion management for IMRT of cervical cancer based on model-predicted cervix-uterus shape and position. Radiother Oncol, 99: 240-5.

30. Jhingran A, Winter K, Portelance L, Miller B, Salehpour M, Gaur R, et al. (2012) A phase II study of intensity modulated radiation therapy to the pelvis for postoperative patients with endometrial carcinoma: radiation therapy oncology group trial 0418. Int J Radiat Oncol Biol Phys, 84: 23-8.

31. Nuyttens JJ, Robertson JM, Yan D, Martinez A (2004) The influence of small bowel motion on both a conventional three-field and intensity modulated radiation therapy (IMRT) for rectal cancer. Cancer Radiother, 8: 297304. 